\title{
A new heterodyne tandem-interferometer for fully interferometric three-dimensional imaging spectrometry
}

\author{
Tetsuya Hashimoto*a, ${ }^{\text {Akiko Hirai }}{ }^{\mathrm{b}}$, Kyu Yoshimori ${ }^{\mathrm{a}}$ \\ ${ }^{a}$ Department of Electrial Engineering and Computer Science, Graduate School of Engineering, Iwate \\ University, Ueda 4-3-5, Morioka, Iwate 020-8551, Japan; ${ }^{b}$ National Institute of Advanced Industrial \\ Science and Technology (AIST), National Metrology Institute of Japan, Tsukuba Central 3, 1-1-1 \\ Umezono, Tsukuba, Ibaraki 305- 8563, Japan
}

\begin{abstract}
We have studied a method to obtain both three-dimensional (3-D) spatial information and spectral information of a usual polychromatic object by a fully passive interferometric technique that is strongly coupled with digital signal processing ${ }^{1-}$ ${ }^{6}$. Our method can be used for a vast range of wavelengths, because no special imaging devices, such as lenses, are required. In addition, coherent light sources are not necessary. A hyperbolic-type volume interferogram ${ }^{6}$ is one type of the volume interferogram, which is used for retrieve the object information, and an interferometer to measure directly this volume interferogram has been introduced ${ }^{3}$.

This paper introduces a method to measure the hyperbolic-type volume interferogram with high sensitivity in fully interferometric 3-D imaging spectrometry. This is accomplished by coupling of interferometer to measure directly the hyperbolic-type volume interferogram and heterodyne detection system ${ }^{7}$ for broadband light. We also report a preliminary experimental result based on the interferometer to measure directly the hyperbolic-type volume interferogram.

Keywords: Digital holography, Interferometric imaging, Spectrometry, Fourier transforms, Imaging spectrometry, Low coherence holography, Signal processing, Heterodyne interferometry
\end{abstract}

\section{INTRODUCTION}

Previously, we have reported experimental results in retrieving the 3-D images for spectral components of monochromatic optical sources ${ }^{2,4}$ and incoherent optical sources ${ }^{3,5,6}$. However, if the same method is applied to a secondary light sources under the illumination of natural light sources, it becomes hard to obtain clear images because of the low contrast of interference fringe patterns produced by the low coherence light. Therefore, it is necessary to improve the sensitivity of measuring the interference pattern. A hyperbolic-type volume interferogram ${ }^{6}$ is one type of a volume interferogram, which is computed from a dataset of originally-measured interference pattern, and the object information can be retrieved from it by proper signal processing. We have been introduced an interferometer ${ }^{3}$ to measure directly this volume interferogram by taking a feature of it.

In this paper, we introduce a method to measure the hyperbolic-type volume interferogram with high sensitivity in fully interferometric 3-D imaging spectrometry. This method uses a tandem-type interferometer, which consists the interferometer to measure directly the hyperbolic-type volume interferogram and an interferometer for heterodyne detection for broadband light ${ }^{7}$. For the heterodyne detection for broadband light, a pair of acousto-optic modulators (AOMs) and spherical mirrors is used. To author's knowledge, this is a first trial to introduce heterodyne technique into the usual homodyne coherence measurement. In addition, we demonstrate the preliminary experiment for retrieval the images of the monochromatic point source based on the directly measurement of the hyperbolic-type volume interferogram.

*tetsuya@ql.cis.iwate-u.ac.jp

ICPS 2013: International Conference on Photonics Solutions, edited by Prathan Buranasiri, Sarun Sumriddetchkajorn, Proc. of SPIE Vol. 8883, 88830T · @ 2013 SPIE · CCC code: 0277-786X/13/\$18 · doi: 10.1117/12.2022104 


\section{PRINCIPLE OF METHOD}

Figure 1 shows a schematic of the interferometer for our method. In the first interferometer, the optical wave propagating from the object under measurement are reflected by the prisms, P and P'. The optical paths of the interferometer are delayed by $d_{1}$. Since two apexes of these prisms are set orthogonally each other, each wave front is reversed vertically or horizontally. We set the lens L1 to project the pinhole along the optical axis onto the apex of $\mathrm{P}^{\prime}$. The pinhole transmits the optical wave along the optical axis. We introduce the displacement $X / 2$ or $Y / 2$ to the prism $\mathrm{P}$ ' or P by $x$ - or $y$ - stage respectively. The optical path difference $Z$ is also introduced by piezoelectric translator (PZT) in addition to $d_{1}$. The optical wave transmitted through the pinhole propagates to a second interferometer for broadband heterodyne detection. It is based on a first interferometer of the tandem-type interferometer shown in Ref. 7, using two pairs of an acoustooptic modulator $(\mathrm{AOM})$ and a spherical mirror. The diffraction angle by the AOM changes with wavelength. However, since the dispersed light is reflected and converged to AOM by spherical mirror, the diffraction angle is compensated. The re-converged optical waves undergo frequency shift by AOMs. Two AOMs are driven by different electrical signal at difference frequency $\Delta \mathrm{f}$. These optical waves generate the heterodyne fringe signal by superposing on the BS when the delay in the second interferometer is approximately equal to the delay $d_{1}$ of the first interferometer. This fringe signal is detected by the photo diode. The signal at $2 \Delta \mathrm{f}$ is taken by using the lock-in amplifier. The dataset obtained with moving the $x$ - and $y$-stage and the PZT consists the hyperbolic-type volume interferogram.

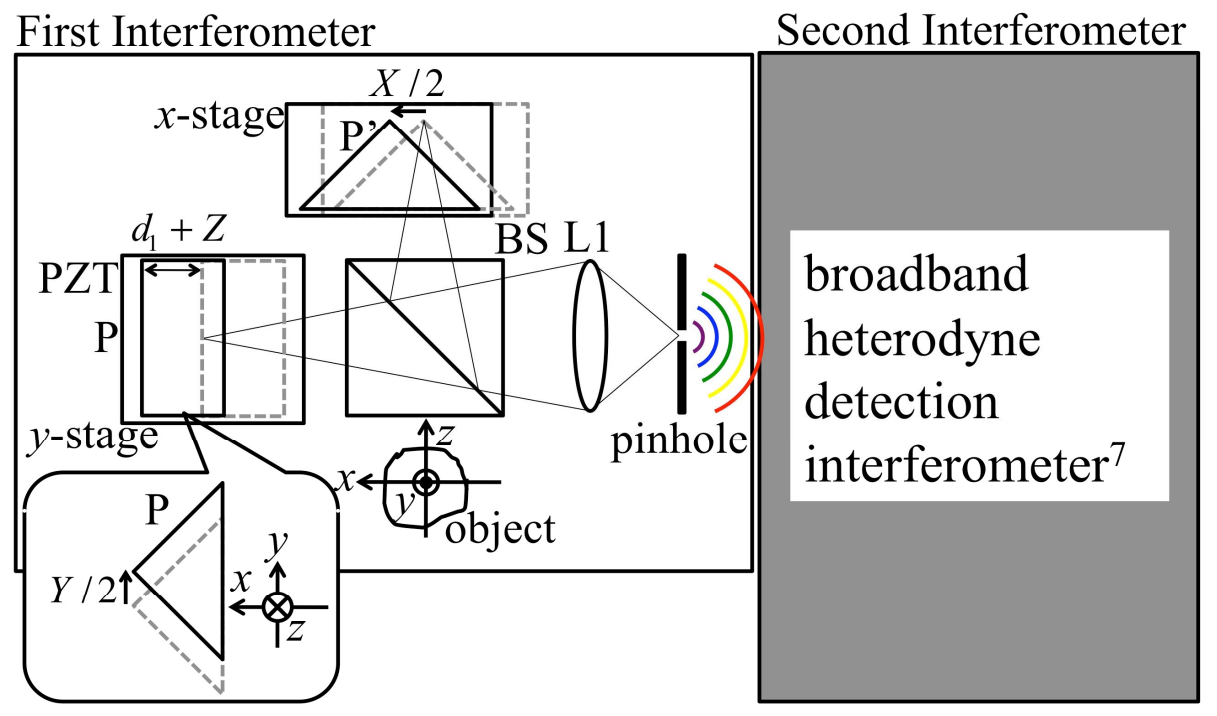

Figure 1. The tandem-type interferometer for fully interferometric 3-D imaging spectrometry

We describe the coherence function $\Gamma\left(\boldsymbol{r}^{\prime}, \boldsymbol{r}\right)$ for the complex optical fields $V(\boldsymbol{r}, t)$ and $V\left(\boldsymbol{r}^{\prime}, t\right)$ at two 3-D positions $\boldsymbol{r}$ and $\boldsymbol{r}^{\prime}$ at the equal time $t$ is defined as $\Gamma\left(\boldsymbol{r}^{\prime}, \boldsymbol{r}\right)=\left\langle V^{*}\left(\boldsymbol{r}^{\prime}, t\right) V(\boldsymbol{r}, t)\right\rangle$, where we suppress $t$ in $\Gamma$ since the optical field is stationary in time. Therefore, the spatial coherence function included in the hyperbolic-type volume interferogram is expressed as $\Gamma\left(X, 0, z_{0} ; 0, Y, z_{0}+Z\right)$. Figure 2 shows an example of the hyperbolic-type volume interferogram. This interferogram is calculated under assumption that the object under measurement is a monochromatic point source of particular wavelength, located at the origin of the Cartesian coordinate system. The fringe patterns across the $X-Y$ surface of this volume interferogram form a hyperbolic as shown in. In addition, we find that the orientations of the curve orientation of the fringe patterns across the $Z-Y$ surface and the $Z-X$ surface are opposite. The retrieval processing of the object information from the hyperbolic-type volume interferogram, measured from the tandem-type interferometer, is the same as the conventional method ${ }^{6}$. 


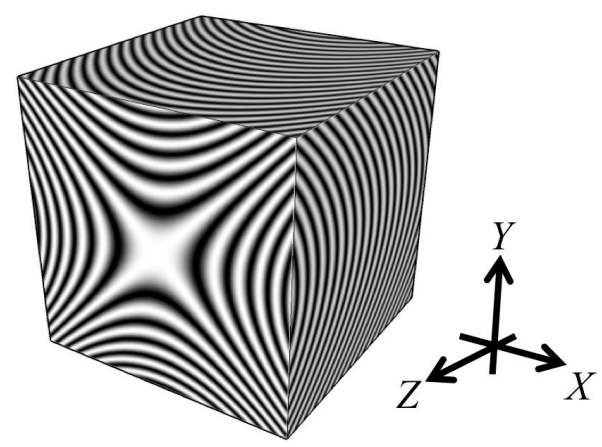

Figure 2. Example of the hyperbolic-type volume interferogram

\section{PRELIMINARY EXPERIMENT}

As a preliminary experiment, we describe the results of retrieve the image for monochromatic point source by using of the first interferometer of Fig. 1. An interferometric measurement using the first interferometer is possible when delay $d_{1}$ of first interferometer is 0 , and a CCD camera is set as substitute for the pinhole A. While the $x$ - and $y$-stages and the PZT are moved stepwise, this CCD camera records one pixel that detects interference intensity along the optical axis. The hyperbolic-type volume interferogram is obtained by rearrangement of the detected interference intensity to the 3-D coordinates $(X, Y, Z)$. In this experiment, A complex hologram is computed based on phase shifting. We may then retrieve the image of the monochromatic point source.

To create the monochromatic point source, we used optical fiber guiding beam from He-Ne Laser. The wavelength of the laser is $543.5 \mathrm{~nm}$. The distance between the light source and the apex of the $\mathrm{P}^{\prime}$ is $62 \mathrm{~mm}$. The numbers of steps and intervals are 32 and $12.9 \mu \mathrm{m}$ for the $x$ - and $y$-stage and 4 and $0.067 \mu \mathrm{m}$ for the PZT. The pixel size of the CCD camera is configured as $12.9 \mu \mathrm{m}$.

Figure 3 shows the phase distribution of a hologram computed from phase shifted interference patterns. It seems that this phase distribution is similar to the $X-Y$ surface of the hyperbolic-type volume interferogram as shown in Fig. 2, although it seems the aberration. Since the infocus distance is different at $X$ - and $Y$-components of the hologram, we find that this hologram includes astigmatism. Figure 4 shows the retrieved images at distance $58 \mathrm{~mm}$ for $X$-component and $34 \mathrm{~mm}$ for $Y$-component. From Fig. 4, we confirmed that the imaging of the monochromatic point source by using of the first interferometer is possible. We consider that the astigmatism of the hologram is caused by asymmetry in the transverse direction of the interferometer.

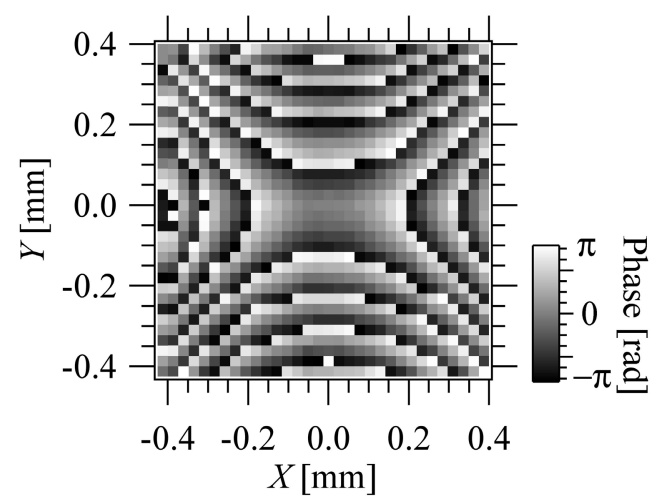

Figure 3. The phase distribution of the hologram 


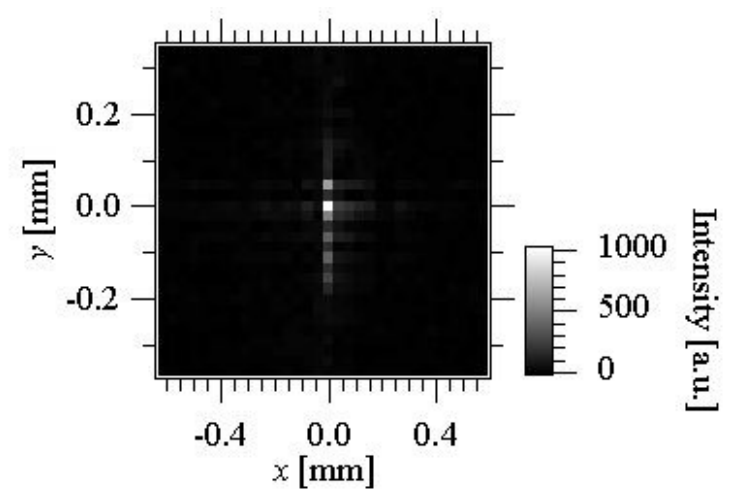

Figure 4. The retrieved images at distance $58 \mathrm{~mm}$ for $X$-component and $34 \mathrm{~mm}$ for $Y$-component

\section{CONCLUSION}

We introduce a method to measure the hyperbolic-type volume interferogram with high sensitivity in fully interferometric 3-D imaging spectrometry. This method uses an interferometer, which couples the interferometer to measure directly the hyperbolic-type volume interferogram and the broadband heterodyne detection interferometer. Preliminary experiment shows the retrieved image of the monochromatic point source based on the direct measurement of the hyperbolic-type volume interferogram and the phase shifting by using only first interferometer.

\section{ACKNOWLEDGMENTS}

This work is supported in part by the Research Foundation for Opto-Science and Technology, and Grants-in-Aid for Scientific Research 25390087 from the Japan Society for the Promotion of Science.

\section{REFERENCES}

[1] Yoshimori K., "Passive digital multispectral holography based on synthesis of coherence function," Proc. SPIE 6252, 625221 (2006).

[2] Sasamoto M. and Yoshimori K., "First experimental report on fully passive interferometric three-dimensional imaging spectrometry," Jpn. J. Appl. Phys. 48, 09LB03 (2009).

[3] Hashimoto T. Hirai A. and Yoshimori K., "Direct measurement of hyperbolic-type volume interferogram," International Workshop on Holography and Related Technologies, 25-26, P02, (2011)

[4] Sasamoto M. and Yoshimori K., "Three-dimensional imaging spectrometry by fully passive interferometry," Opt. Rev. 19, 29-33 (2012)

[5] Teeranutranont S. and Yoshimori K., "Digital holographic three-dimensional imaging spectrometry," Appl. Opt. 52, A388-A396 (2013).

[6] Hashimoto T. Hirai A. and Yoshimori K., "Fully interferometric three-dimensional imaging spectrometry using hyperbolc-type volume interferogram," Appl. Opt. 52, 1497-1504 (2013).

[7] Hirai A. and Matsumoto H., "High-sensitivity surface-profile measurements by heterodyne white-light interferometer," Opt. Eng. 40, 387-391 (2001). 